\title{
The secondary minimum in YY Herculis: Evidence for a tidally distorted giant
}

\author{
J. Mikołajewska ${ }^{1}$, E. A. Kolotilov ${ }^{2}$, S. Yu. Shugarov ${ }^{2,3}$, and B. F. Yudin ${ }^{2,3}$ \\ 1 N. Copernicus Astronomical Center, Bartycka 18, 00716 Warsaw, Poland \\ 2 Sternberg Astronomical Institute, Universitetskii pr. 13, Moscow 119899, Russia \\ 3 Isaac Newton Institute of Chile, Moscow Branch, Russia
}

Received 28 March 2002 / Accepted 11 June 2002

\begin{abstract}
We present and analyze quiescent $U B V R I$ light curves of the classical symbiotic binary YY Her. We show that the secondary minimum, which is clearly visible only in the quiescent $V R I$ light curves, is due to ellipsoidal variability of the red giant component. Our simple light curve analysis, by fitting of the Fourier cosine series, resulted in a self-consistent phenomenological model of YY Her, in which the periodic changes can be described by a combination of the ellipsoidal changes and a sinusoidal changes of the nebular continuum and line emission.
\end{abstract}

Key words. stars: binaries: eclipsing - binaries: symbiotic - stars: fundamental parameters - stars: individual: YY Her

\section{Introduction}

Symbiotic stars are long-period interacting binaries made up of an evolved red giant and a hot companion surrounded by an ionized nebula. The hot component in the vast majority of systems seems to be a luminous $\left(\sim 1000 L_{\odot}\right)$ and hot $\left(\sim 10^{5} \mathrm{~K}\right)$ white dwarf powered by thermonuclear burning of the material accreted from its companion's wind. Depending on the accretion rate, these systems can be either in a steady burning configuration or undergo hydrogen shell flashes, which in many cases can last for decades and even centuries due to low mass of the white dwarf. The latter can be the case for non-eruptive symbiotics (such as RW Hya or V443 Her), in which the hot components maintain roughly constant luminosity and temperature for many decades (Mikołajewska 1997). In many systems, however, the hot components show multiple outburst activity with timescales of a few/several years, which cannot be simply accounted for by the thermonuclear models. Among them, $\mathrm{Z}$ And is one of the best studied (e.g. Mikołajewska \& Kenyon 1996, and references therein), and the symbiotic stars with multiple outburst activity are often referred to as Z And-type systems.

The photometric history of YY Her since 1890 has been studied in detail by Munari et al. (1997a, hereafer M97a) who revealed, in addition to $\mathrm{Z}$ And-type multiple outburst activity, a periodic fluctuations with $P=590^{\mathrm{d}}$ and a visual amplitude of $\lesssim 0.3^{\mathrm{m}}$. The orbital nature of this periodicity has been confirmed by subsequent $U B V$ photometric observations (Munari et al. 1997b; Tatarnikova et al. 2001, hereafter M97b and T01,

Send offprint requests to: J. Mikołajewska,

e-mail: mikolaj@camk.edu.pl respectively). In particular, the photometric and spectroscopic study of YY Her during the major outburst in 1993 and its decline (Tatarnikova et al. 2000, hereafer T00; T01) has shown that the minima are due to obscuration of the hot component and the ionized nebula. The return to quiescence in 1997/98 was accompanied by significant changes in the shape of light curves, and in particular by the appearance of a secondary minumum in the VRI light curves (T01; Hric et al. 2001, hereafter H01). T01 interpreted the secondary minimum in terms of ellipsoidal changes of the red giant, whereas H01 argued that the secondary minimum is caused by an eclipse of the red giant by a circumstellar envelope around the hot component.

In this paper we present new $U B V R I$ photometric observations obtained during the secondary minimum in 2001, and demonstrate that the quiescent light curves of YY Her can be succesfully reproduced by a combination of ellipsoidal changes of the cool giant and sinusoidal changes of the nebular continuum and line emission.

\section{Observations}

$U B V R^{\prime} I^{\prime}$ photometry was obtained with the $0.6-\mathrm{m}$ and $1.25-\mathrm{m}$ telescopes at the Crimean Station of the Sternberg Astronomical Institute (SAI). For $U B V$ bands (Table 1) the 2-channel photometer with an S-20 photomultiplier was used, whereas a CCD matrix was the detector for $B V R^{\prime} I^{\prime}$ bands (Table 2; see below for the definition of $R^{\prime} I^{\prime}$ ). The uncertainty on individual measurements does not exceed 0.03 mag. The $U B V$ bands correspond to Johnson's broad-band system. The instrumental $r i$ bands have the effective wavelengths, $\lambda_{r} \approx 7000 \AA$ and $\lambda_{i} \approx 9000 \AA$, and the band widths 
Table 1. $U B V$ photometry of YY Her.

\begin{tabular}{lccc}
\hline \hline $\mathrm{JD}(2440000+)$ & $U$ & $B$ & $V$ \\
\hline 12047 & 13.73 & 14.24 & 12.95 \\
12048 & 13.66 & 14.21 & 12.97 \\
12055 & & 14.26 & 13.10 \\
12106 & 13.66 & 14.30 & 13.16 \\
12115 & 13.70 & 14.32 & 13.22 \\
12135 & 13.38 & 14.28 & 13.32 \\
12138 & 13.62 & 14.42 & 13.39 \\
12168 & 13.38 & 14.27 & 13.18 \\
12172 & 13.46 & 14.20 & 13.14 \\
12191 & 13.43 & 14.17 & 13.06 \\
12195 & 13.50 & 14.13 & 13.04 \\
12202 & 13.38 & 14.13 & 13.01 \\
12212 & 13.33 & 14.07 & 12.96 \\
12223 & 13.35 & 14.03 & 12.86 \\
\hline
\end{tabular}

Table 2. $B V R^{\prime} I^{\prime}$ photometry of YY Her.

\begin{tabular}{lcccc}
\hline \hline $\mathrm{JD}(2440000+)$ & $B$ & $V$ & $R^{\prime}$ & $I^{\prime}$ \\
\hline 11865 & & 13.59 & 11.76 & 10.06 \\
11866 & & 13.59 & 11.74 & 10.05 \\
11867 & & 13.64 & 11.76 & 10.09 \\
11868 & & 13.64 & 11.73 & 10.08 \\
11887 & 14.87 & 13.40 & 11.58 & 10.00 \\
11888 & 14.86 & 13.40 & 11.59 & 10.02 \\
11952 & & 13.14 & 11.54 & 10.10 \\
12029 & & 12.91 & 11.35 & 9.91 \\
12033 & & 12.94 & 11.33 & 9.90 \\
12090 & & 12.99 & 11.33 & 9.93 \\
12162 & 14.27 & 13.14 & 11.51 & 10.08 \\
12173 & 14.26 & 13.17 & 11.46 & 10.05 \\
12176 & & 13.08 & 11.43 & 10.03 \\
12178 & & 13.08 & 11.42 & 10.01 \\
12188 & & 13.02 & 11.39 & 9.99 \\
12191 & 14.21 & 13.02 & 11.36 & 9.97 \\
12247 & 14.04 & 12.95 & 11.33 & 9.93 \\
\hline
\end{tabular}

$\Delta \lambda_{r} \approx 2400 \AA$ and $\Delta \lambda_{i} \approx 3000 \AA$, respectively, and they are different from any commonly used photometric system. The $r i$ observations were reduced to Johnson's RI magnitudes using the standard stars from M 67 (Mendoza 1967). In the case of stars with a combination spectra such as YY Her, a transformation of the instrumental magnitudes to the standard system may be not as precise as in the case of normal stars. For this reason, we refer to these magnitudes as $R^{\prime} I^{\prime}$. HD 168957 ( $U=6.35$, $B=6 \cdot 91, V=7 \cdot 01)$, and the star designated as $\mathrm{G}(V=13 \mathrm{~m} .08$, $\left.R^{\prime}=12^{\mathrm{m}} \cdot 44, I^{\prime}=11^{\mathrm{m}} \cdot 94\right)$ on the finding chart in M97a) were adopted as the comparison stars.

Our data have been combined with published $U B V$ photometry (T01, and references therein) as well as $B_{\mathrm{c}} V_{\mathrm{c}} R_{\mathrm{c}} I_{\mathrm{c}}$ one performed in the modified Johnson-Kron-Cousins system by $\mathrm{H} 01$. The resulting light curves are shown in Fig. 1. The $B_{\mathrm{c}} V_{\mathrm{c}} R_{\mathrm{c}} I_{\mathrm{c}}$ magnitudes in Fig. 1 were transformed to our system assuming $B=B_{\mathrm{c}}+0.3, V=V_{\mathrm{c}}+0.2, R^{\prime}=R_{\mathrm{c}}-0.2$, and $I^{\prime}=I_{\mathrm{c}}-0.52$, respectively.

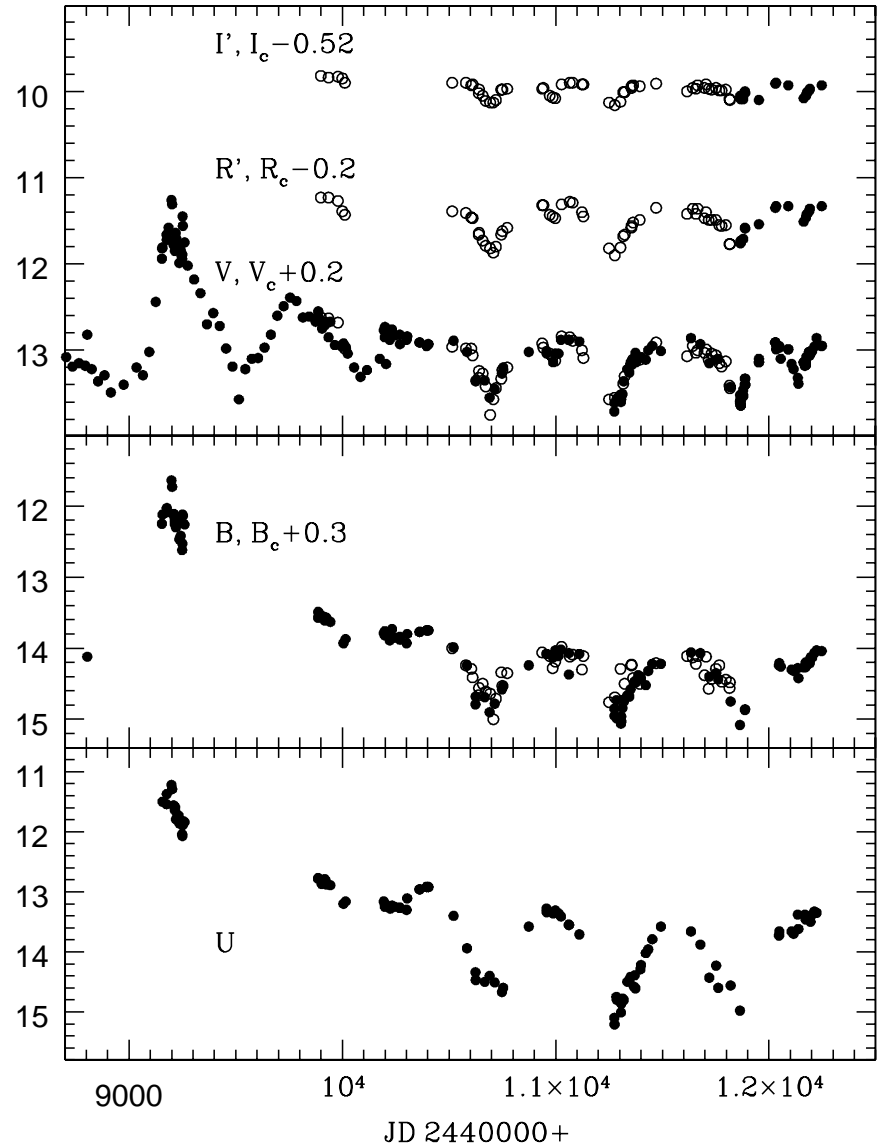

Fig. 1. The UBVRI photometry of YY Her in 1993-2001. Closed symbols represent our $U B V R^{\prime} I^{\prime}$ data (this study; M97a; T00; T01), and open symbols the $B_{\mathrm{c}} V_{\mathrm{c}} R_{\mathrm{c}} I_{\mathrm{c}}$ data of H01) transformed to our system (see text).

\section{Analysis}

The most prominent features of the $U B V$ light curves of YY Her (Fig. 1) are deep eclipses and the large outburst that started in 1993. The $R_{\mathrm{c}} I_{\mathrm{c}} / R^{\prime} I^{\prime}$ light curves cover the late decline and return to quiecence, and they show the characteristic double hump. The secondary minimum is also clearly visible in the quiescent $V$ light curve as well as the most recent $B$ data.

In our analysis, we will focus on the quiescent light curves. Figure 2 shows the UBVRI magnitudes folded over the last three orbital periods. We have adopted the ephemeris,

$\operatorname{Min}=\mathrm{JD} 2450686.2( \pm 2.1)+589.5( \pm 0.3) \times E$

derived from 13 individual minima estimates (by a parabolic fit) in published VRI light curves (M97a; M97b; T00; T01; H01). The salient characteristic of the quiescent light curves, namely: (i) nearly sinusoidal shape of the $U$ light curves; (ii) a flat toped $B$ light curve; and (iii) the two minima of almost comparable depth in $I$ light, combined with the fact that the quiescent, ultraviolet + optical, spectra of YY Her can be satisfactorily matched with a three-component model composed of a cool giant dominating the red spectral range, a gaseous nebula dominating the near $U V$ range, and the hot component predominant in the short $U V$ flux (T00), led us to a simple phenomenological model in which the light changes 


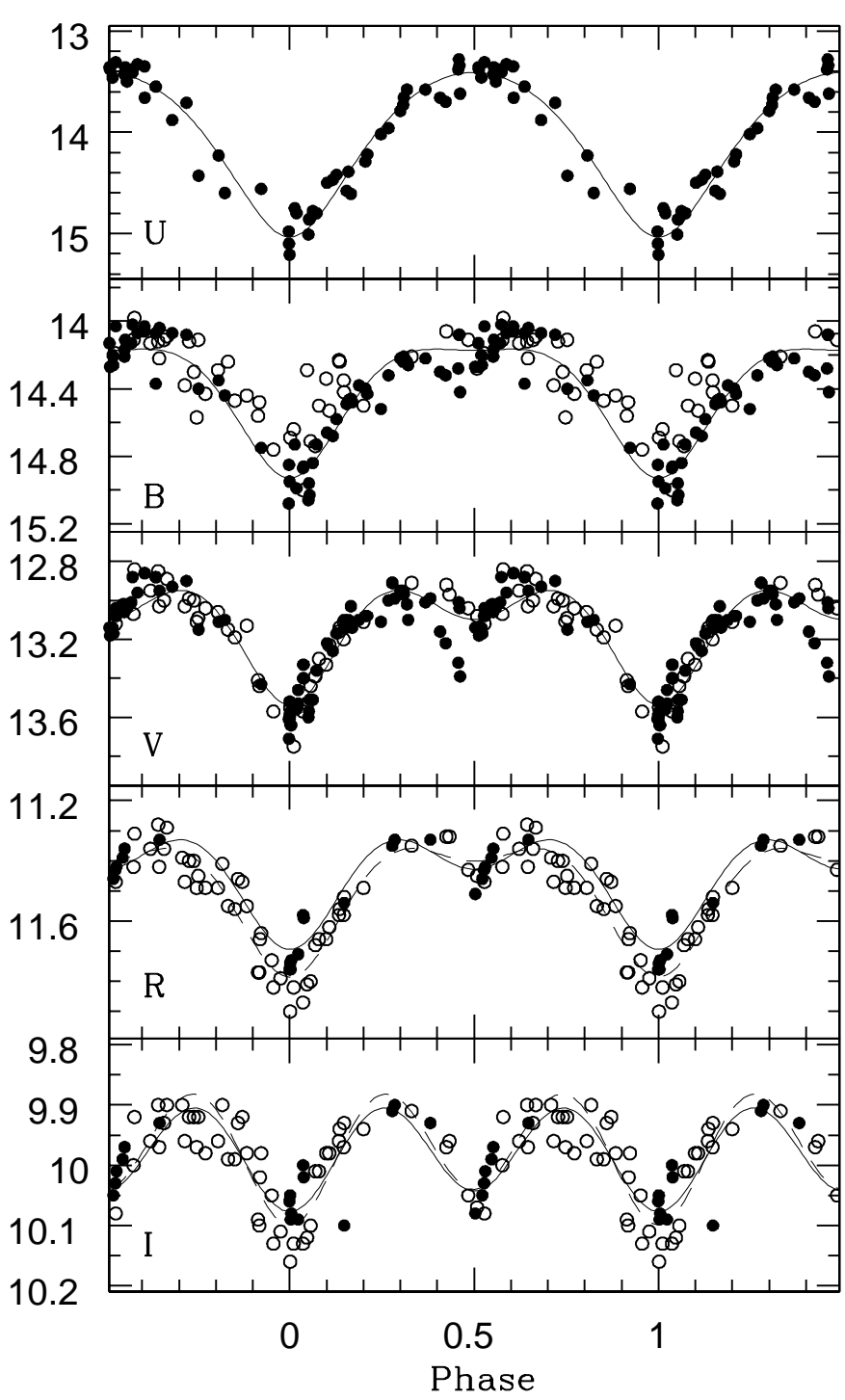

Fig. 2. Phased quiescent light curves of YY Her. The same symbols as in Fig. 1 are used. Our best fit models (Table 3) are plotted as solid lines (our data) and dashed lines (H01 data), respectively.

are described by a combination of variable nebular emission and ellipsoidal changes of the red giant.

Each light curve has been converted to fluxes, $F_{\lambda}=$ $10^{-0.4 m_{\lambda}}$, and then independently fitted by the Fourier cosine series

$F_{\lambda}=F_{\lambda, 0}-A_{\mathrm{n}, \lambda} \cos \phi-A_{\mathrm{g}, \lambda} \cos 2 \phi$.

The results of our fits to the $U B V R^{\prime} I^{\prime}$ (our data) and $R_{\mathrm{c}} I_{\mathrm{c}}$ (H01 data) light curves are summarized in Table 3, Cols. 24. In the case of $U$ data, the best fit is obtained with the $\cos \phi$ term only. This indicates that the contribution of the giant to the $U$ light is negligible, and that all changes can be attributted to the nebular emission. Assuming that the amplitude of the nebular emission changes is the same at all wavelenghts, $\left(F_{\mathrm{n}, \lambda}^{0}+A_{\mathrm{n}, \lambda}\right) /\left(F_{\mathrm{n}, \lambda}^{0}-A_{\mathrm{n}, \lambda}\right) \approx\left(F_{\mathrm{U}, 0}+A_{\mathrm{U}}\right) /\left(F_{\mathrm{U}, 0}-A_{\mathrm{U}}\right)=C \sim 4.5$, we can separate the contributions of the nebular and the giant components to the constant $F_{\lambda, 0}$. The resulting charateristics of the nebular source (Cols. 5-7) and the red giant (Cols. 8-10) are also given in Table 3 .
The maximum, $F_{\lambda, \max }(\phi=0.5)=F_{\mathrm{n}, \lambda}^{0}+A_{\mathrm{n}, \lambda}$, fluxes of the nebular component have been calculated using the zeroth magnitude star fluxes from Straižys (1992). The $U B V R^{\prime} I^{\prime}$ and $R_{\mathrm{c}} I_{\mathrm{c}}$ magnitudes corresponding to these maximum fluxes have been also calculated. We note here that the broad-band magnitudes, and the resulting fluxes may be significantly afftected by emission lines. Using the quiescent optical/red spectra of YY Her (T00; T01), we have calculated the contribution of the emission lines to the observed $B V R^{\prime}$ magnitudes as $\Delta m \gtrsim 0.15 / 0.02 / 0.08 \mathrm{mag}$, respectively (this contribution can be, in fact, even higher because we have not included faint lines). Using these estimates, we have found that the nebular line emission is responsible for at least 30\% ( $B$ band), $7 \%$ $(V)$ and $50 \%\left(R^{\prime}\right)$ of the total nebular emission fluxes, and the corresponding magnitudes, given in the 5th and 6th columns of Table 3. Finally, the maximum emission measure of the nebular continuum ( 7 th column of Table 3 ) has been estimated from the maximum, $F_{\lambda \text {,max }}$, fluxes of the nebular component corrected for the contribution from emission lines and the interstellar reddening $E_{B-V}=0.2(\mathrm{M} 97 \mathrm{~b})$,

$E M=n_{\mathrm{e}}^{2} V / 4 \pi d^{2}=F_{\lambda, \max } / \epsilon_{\lambda}^{\mathrm{bf}+\mathrm{ff}}$.

The volume emission coefficients, $\epsilon_{\lambda}^{\mathrm{bf}+\mathrm{ff}}$, have been estimated using our $U B V R^{\prime} I^{\prime}$ filter transmission functions and $\mathrm{bf}+\mathrm{ff}$ continuum emission coefficients (case $\mathrm{B}$ recombination) from Pottash (1984). The $E M$ values derived for each band agree with each other to within a factor of 2 , and they cluster around $E M \sim 2 \times 10^{14}$ and $\sim 3 \times 10^{14} \mathrm{~cm}^{-5}$ for $T_{\mathrm{e}}=10^{4}$ and $2 \times 10^{4} \mathrm{~K}$, respectively.

The nebular emission measure derived from our light curve analysis requires a Lyman continuum luminosity of $\sim 6-5 \times$ $10^{45}\left(d / 1 \mathrm{kpc}^{2}\right.$ phot s$^{-1}$ for $T_{\mathrm{e}}=10^{4}-2 \times 10^{4} \mathrm{~K}$, which corresponds to a hot component with $T_{\mathrm{h}} \sim 10^{5} \mathrm{~K}$ and $L_{\mathrm{h}} \sim$ $80(d / 1 \mathrm{kpc})^{2} L_{\odot}$. This can be compared with independent estimates for the quiescent $L_{\mathrm{h}}$. In particular, the bolometric fluxes for the hot component given by M97b and T00 correspond to $L_{\mathrm{h}} \sim(30-60)(d / 1 \mathrm{kpc})^{2} L_{\odot}$.

The $U B V R^{\prime} I^{\prime}$ and $R_{\mathrm{c}} I_{\mathrm{c}}$ magnitudes of the cool component have been calculated using $m_{0}=-2.5 \log F_{\mathrm{g}, \lambda}^{0}$, $m_{\max }=-2.5 \times \log \left(F_{\mathrm{g}, \lambda}^{0}+A_{\mathrm{g}, \lambda}\right)$, and $m_{\min }=-2.5 \log \left(F_{\mathrm{g}, \lambda}^{0}-A_{\mathrm{g}, \lambda}\right)$, where $F_{\mathrm{g}, \lambda}^{0}$ is the cool component contribution to the constant $F_{\lambda, 0}$. The colours derived from these magnitudes, $B-V=1.75 / 1.77 / 1.73, V-R^{\prime}=1.81 / 1.73 / 1.92$ and $V-I^{\prime}=3.45 / 3.33 / 3.61$ representing the average, maximum and minimum values, respectively, are in excellent agreement with those expected for a moderately reddened M3-M4 giant. Moreover, they indicate somewhat higher temperature (earlier spectral type) at maxima ( $\phi=0.25$ and 0.75$)$ than that at minima $(\phi=0$ and 0.5$)$ as expected for a tidally distorted giant.

Assuming the Roche-lobe filling factor $R_{\mathrm{g}} / R_{\mathrm{L}} \approx 1$, the gravity-darkening exponent, $\alpha=0.32$ (convective envelope), and the system inclination $i \sim 70-90^{\circ}$, the amplitude of the cool component changes in $I^{\prime}$ is consistent with a mass ratio $q=M_{\mathrm{g}} / M_{\mathrm{h}} \sim 2-3$. This estimate is based on synthetic light curves calculated using the Wilson-Devinney (WD) code (see Belczyński \& Mikołajewska 1998 for details). The amplitudes in $R, V$ bands, however, require a much higher value 
Table 3. The best fit parameters for YY Her.

\begin{tabular}{|c|c|c|c|c|c|c|c|c|c|}
\hline \multirow[t]{2}{*}{ Filter } & \multicolumn{3}{|c|}{ Best fit parameters } & \multicolumn{3}{|c|}{ Hot nebular source } & \multicolumn{3}{|c|}{ Cool giant } \\
\hline & $F_{\lambda, 0}^{1}$ & $A_{\mathrm{n}, \lambda}^{1}$ & $A_{\mathrm{g}, \lambda}^{1}$ & $F_{\lambda, \max }^{2}$ & $m_{\max }[\mathrm{mag}]$ & $E M^{3}$ & $m_{0}[\mathrm{mag}]$ & $m_{\max }[\mathrm{mag}]$ & $m_{\min }[\mathrm{mag}]$ \\
\hline$U$ & $2.65 \pm 0.05$ & $1.68 \pm 0.06$ & 0 & $17.8 \pm 0.4$ & $13.41 \pm 0.03$ & $1.5 / 2.7$ & & & \\
\hline$B$ & $1.77 \pm 0.02$ & $0.54 \pm 0.03$ & $0.17 \pm 0.03$ & $8.6 \pm 0.3$ & $14.64 \pm 0.06$ & $3.0 / 2.4$ & $15.21 \pm 0.06$ & $15.03 \pm 0.08$ & $15.43 \pm 0.11$ \\
\hline V & $5.64 \pm 0.05$ & $0.96 \pm 0.06$ & $0.84 \pm 0.07$ & $9.0 \pm 0.4$ & $14.02 \pm 0.06$ & $2.8 / 3.2$ & $13.46 \pm 0.04$ & $13.26 \pm 0.05$ & $13.70 \pm 0.07$ \\
\hline$R^{\prime 4}$ & $26.4 \pm 0.4$ & $2.9 \pm 0.4$ & $2.5 \pm 0.4$ & $12.4 \pm 0.9$ & $12.82 \pm 0.14$ & $2.0 / 2.9$ & $11.65 \pm 0.03$ & $11.53 \pm 0.04$ & $11.78 \pm 0.05$ \\
\hline$R_{\mathrm{c}}^{5}$ & $26.5 \pm 0.2$ & $3.4 \pm 0.3$ & $1.7 \pm 0.3$ & $19.7 \pm 0.9$ & $12.64 \pm 0.09$ & $3.2 / 4.5$ & $12.00 \pm 0.03$ & $11.89 \pm 0.04$ & $12.13 \pm 0.05$ \\
\hline$I^{\prime 4}$ & $101.9 \pm 0.9$ & $1.5 \pm 0.7$ & $7.2 \pm 1.1$ & $3.0 \pm 0.9$ & $13.5 \pm 0.5$ & $1.6 / 2.0$ & $10.01 \pm 0.02$ & $9.93 \pm 0.03$ & $10.09 \pm 0.04$ \\
\hline$I_{\mathrm{c}}^{5}$ & $63.5 \pm 0.6$ & $1.6 \pm 0.8$ & $5.4 \pm 0.8$ & $4.9 \pm 1.5$ & $13.5 \pm 0.5$ & $2.5 / 3.0$ & $10.54 \pm 0.03$ & $10.44 \pm 0.04$ & $10.64 \pm 0.05$ \\
\hline
\end{tabular}

1 in units of $10^{-6} ;{ }^{2}$ in units of $10^{-15} \mathrm{erg} \mathrm{s}^{-1} \mathrm{~cm}^{-2} \AA^{-1} ;{ }^{3}$ maximum emission measure in units of $10^{14} \mathrm{~cm}^{-5}$, for $T_{\mathrm{e}}=10^{4} / 2 \times 10^{4} \mathrm{~K}$; ${ }^{4}$ our $R^{\prime} / I^{\prime}$ data; ${ }^{5} R_{\mathrm{c}} / I_{\mathrm{c}}$ from $\left.\mathrm{H} 01\right)$.

of $\alpha \gtrsim 1$. Similar effect was found in $\mathrm{T} \mathrm{CrB}$, and it is most likely due to the blackbody approximation for wavelength dependence in the WD code used to calculate the synthetic light curves (Belczyński \& Mikołajewska 1998). This assumption is probably not valid in the case of M-type stars with strong contribution of $\mathrm{TiO}$ bands in the optical and red part of the spectrum. Recently, Orosz \& Hauschildt (2000) have demonstrated that, in general, the light curves for tidally distorted cool giants computed using the NEXTGEN model atmospheres for cool giants have deeper minima in the optical range then their blackbody counterparts.

Finally, we note that the amplitude of ellipsoidal variability is a strong function of the Roche-lobe filling factor, $\Delta m \propto$ $\left(R_{\mathrm{g}} / R_{\mathrm{L}}\right)^{3}$ (e.g. Hall 1990). The lower limit for the cool component changes set by the uncertainty of our fits (Table 3 ) then requires $R_{\mathrm{g}} / R_{\mathrm{L}} \gtrsim 0.9$ for $q \gtrsim 1$ and $i \gtrsim 70^{\circ}$.

The presence of a Roche-lobe filling giant provides an opportunity to estimate the distance $d$ to YY Her. The Roche lobe radius is $R_{\mathrm{L}} / a \approx 0.44 \pm 0.05$ for the mass ratio of $q=M_{\mathrm{g}} / M_{\mathrm{h}} \sim 1-3$ (Paczyński 1971). Our orbital period requires the binary separation, $a \approx 1.85 \pm 0.15$ a.u. for a reasonable total mass of the system, $M_{\mathrm{g}}+M_{\mathrm{h}} \sim 2-3 M_{\odot}$ (e.g. Mikołajewska 1997), so $R_{\mathrm{L}} \approx 0.8 \pm 0.2$ a.u. Using the BarnesEvans relation (Cahn 1980),

$F_{\mathrm{K}}=4.2207-0.1 K-0.5 \log s$,

with the $K$ surface brightness, $F_{\mathrm{K}}=3.84$ (M4 III), and $K_{0}=$ 7.9 , we estimate the giant's angular diameter, $s=0.152$ mas, and $d=10 \pm 3 \mathrm{kpc}$. Such a distance implies a very high $\sim 3 \mathrm{kpc}$ height above galactic plane and locates YY Her in galactic halo.

\section{Concluding remarks}

Our very simplistic light curve analysis, namely independent fitting of the Fourier cosine series to the quiescent $U B V R^{\prime} I^{\prime}$ light curves, resulted in a self-consistent phenomenological model of YY Her, in which the periodic changes can be described by variations of the nebular emission along the orbit (the $\cos \phi$ term) combined with the ellipsoidal variability of the red giant (the $\cos 2 \phi$ term).

YY Her is not the only symbiotic system with the secondary minima. Similar light curve behaviour is observed in
CI Cyg, whose quiescent UBVRI light curves (Mikołajewska 2001) are almost identical with the light curves shown in Fig. 2. The secondary minima are also present in quiescent near infrared light curves of BF Cyg (Mikołajewska et al. 2002).

We also think that the alternative interpretation of the secondary minimum in terms of the eclipse of the cool giant by the circumstellar envelope of the hot component (H01) is implausible. First, it cannot be the ionized nebular envelope because to cause eclipses it must be optically thick in the optical/red continuum whereas the presence of a prominent Balmer jump indicates that it is not. Second, to account for the amplitudes of the secondary minimum, it must screen $\sim 20 \%$ of the red giant's projected surface. Such a big and hot screen, however, should dominate the quiescent optical continuum, which is not observed. Finally, we can also exclude eclipses of the cool giant by an accretion disc. A geometrically and optically thick disc seen nearly edge-on could mimic a cool source, and screen significant part of the giant's surface. Such a disc, however, would also completely hide the hot white dwarf whereas the quiescent shortwavelength IUE spectrum clearly shows a hot continuum (M97b). Similarly, a hot rising continuum is present in quiescent shortwavelength IUE spectra of CI Cyg and BF Cyg.

One of fundamental questions in relation of symbiotic binaries is the mechanism that powers the $\mathrm{Z}$ And-type multiple outburst activity observed in many classical symbiotic systems. The quiescent spectra of these systems can mostly be fitted by a hot stellar source (most likely a white dwarf powered by thermonuclear burning of the material accreted from its companion) and its ionizing effect on a nebula (e.g. Mürset et al. 1991; M97b, Mikołajewska \& Kenyon 1996). Their outburst activity, however, with time scales of a few/several years cannot be simply accounted by the thermonuclear models. A promising interpretation of this activity involves changes in mass transfer and/or accretion disc instabilities. Detection of an ellipsoidal hot continuum source during outbursts of CI Cyg, AX Per, YY Her, AS 338 and other Z And-type systems suggests the presence of an optically thick accretion disc (Mikołajewska \& Kenyon 1992; Esipov et al. 2000; T00; Mikołajewska et al. 2002) which strongly supports this interpretation.

Related to this problem is the process of mass transfer - Roche lobe overflow or stellar wind - and the possibility of an accretion disc formation. The presence of secondary 
minima in the light curves of YY Her, apparently due to ellipsoidal changes of the red giant, as well as in a few other similar systems provides important clues. Although it is very premature to claim that all symbiotic systems with the Z And-type activity do have tidally distorted giants, and - at least during active phase - accretion discs, whereas the non-eruptive systems (such as RW Hya) do not, the former is certainly the case for YY Her, CI Cyg and a few other active systems.

Systematic searches for ellipsoidal variations in both active and non-eruptive symbiotic stars are necessary to address the problem, and confirm or exclude tidally distorted giants. The observations must be, however, carried in the red and near-IR range where the cool giant dominates the continuum light. We also note that any optical/red light curve analysis must be supported by spectroscopic information about contamination of the broad-band photometry by the nebular component.

Acknowledgements. This research was partly supported by KBN research grant No. 5P03D 019 20, and RFBR grant Nos. 02-02-16235, 02-02-16462 and 02-02-17524.

\section{References}

Belczyński, K., \& Mikołajewska, J. 1998, MNRAS, 296, 77

Cahn, J. H. 1980, Space Sci. Rev., 27, 457

Esipov, V. F., Kolotilov, E. A., Mikołajewska, J., et al. 2000, Astron. Lett., 26, 162

Hall, D. S. 1990, AJ, 100, 554
Hric, L., Petrik, K., Niarchos, P., Velic, Z., \& Gális, R. 2001, IBVS, 5046 (H01)

Mendoza, E. E. 1967, Boletin de Los Observatorios Tonantzinta y Tacubaya, 4(29), 149

Mikołajewska, J. 1997, in Physical processes in symbiotic binaries, ed. J. Mikołajewska (Copernicus Found. for Polish Astronomy, Warsaw), 3

Mikołajewska, J. 2001, in Small-Telescope Astronomy on Global Scales, ed. B. Paczyński, W. P. Chen, \& C. Lemme, ASP Conf. Ser., 246, 167

Mikołajewska, J., \& Kenyon, S. J. 1992, MNRAS, 256, 177

Mikołajewska, J., \& Kenyon, S. J. 1996, AJ, 112, 1659

Mikołajewska, J., Kolotilov, E. A., Shenavrin, V. I., \& Yudin, B. F. 2002, in The Physics of Cataclysmic Variables and Related Objects, ed. B. T. Gansicke, K. Bauermann, \& K. Reinsch, ASP Conf. Ser., 261, 645

Munari, U., Rejkuba, M., Hazen, M., et al. 1997a, A\&A, 323, 113 (M97a)

Munari, U., Kolotilov, E. A., Popova, A. A., \& Yudin, B. F. 1997b, Astron. Rep., 41, 802 (M97b)

Mürset, U., Nussbaumer, H., Schmid, H. M., \& Vogel, M. 1991, A\&A, 248,458

Orosz, J. A., \& Hauschildt, P. H. 2000, A\&A, 364, 2650

Paczyński, B. 1971, ARA\&A, 9, 183

Pottash, S. R. 1984, Planetary Nebulae (D. Reidel), 72

Straižys, V. 1992, Multicolor Stellar Photometry, Pachart Astron. Astrophys. Ser. 15 (Tucson), 297

Tatarnikova, A. A., Rejkuba, M., Buson, L. M., et al. 2000, Astron. Rep., 44, 190 (T00)

Tatarnikova, A. A., Esipov, V. F., Kolotilov, E. A., et al. 2001, Astron. Lett., 27, 703 (T01) 